\title{
PREVISÃO DE DEMANDA DE ORDENS DE SERVIÇO EMERGENCIAIS UTILIZANDO ANÁLISE DE SÉRIES TEMPORAIS
}

\author{
Pétrick Maurat da Conceição ${ }^{1}$ \\ petrick_maurat@id.uff.br \\ Mozart Caetano Heymann ${ }^{1}$ \\ mozart.mch@gmail.com \\ Vitor Hugo Ferreira ${ }^{1}$ \\ vitor@vm.uff.br \\ Helder Gomes Costa ${ }^{1}$ \\ helder.hgc@gmail.com
}

\begin{abstract}
${ }^{1}$ Universidade Federal Fluminense - Pós-Graduação Mestrado e Doutorado Rua Passo da Pátria, 156 Bloco D -São Domingos, Niterói - RJ - CEP 24210-240

\section{RESUMO}

A previsão da demanda futura é de vital importância para a maioria das empresas. Ela permite que a companhia se prepare para o futuro de forma mais eficaz e aprimore suas decisões estratégicas. Nas visão das concessionárias de energia elétrica a previsão da demanda futura é essencial para manter a qualidade de serviço e atendimento, garantindo o fornecimento de energia elétrica aos seus consumidores e cumprimento dos requisitos da ANEEL. Este trabalho tem como objetivo apresentar a resolução do problema da previsão de demanda de OS emergenciais atráves da utilização de séries temporais. O caso estudado é referente à previsão de OS emergenciais horárias da ENERGISA TOCANTINS DISTRIBUIDORA DE ENERGIA S/A através suavização exponencial.

Palavra-chave: Previsão de Demanda; Séries Temporais; Holt-Winters; Ordens de Serviço Emergenciais.

\begin{abstract}
Demand forecasting is of great importance for most businesses. It allows the company to prepare for the future in a more efficient way and improve its strategic decisions. On the energy distribution company point of view the demand forecast is essential to keep the quality of the customer service, guaranteeing the supply of electricity to its consumers and the fulfilment of ANEEL requirements. The case of this study refers to the hourly forecast of emergency service orders from ENERGISA TOCANTINS DISTRIBUIDORA DE ENERGIA S/A using exponential smoothing methods.
\end{abstract}

Keywords: Demand Forecast; Time Series; Holt-Winters; Emergency Service Orders.

\section{Como Citar:}

CONCEIÇÃO, Pétrick Maurat da; HEYMANN, Mozart Caetano; FERREIRA, Vitor Hugo; COSTA, Helder Gomes. Previsão de Demanda de Ordens de Serviço Emergenciais utilizando Análise de Séries Temporais. In: SIMPÓSIO DE PESQUISA OPERACIONAL E LOGÍSTICA DA MARINHA, 19., 2019, Rio de Janeiro, RJ. Anais [.. . ]. Rio de Janeiro: Centro de Análises de Sistemas Navais, 2019. 


\section{INTRODUÇÃO}

As concessionárias de energia elétrica precisam garantir a qualidade do serviço prestado, e um dos problemas mais significativos que afeta esta qualidade do serviço é a interrupção no fornecimento, muitas vezes relacionada com ocorrências emergenciais as quais requerem a intervenção humana para restabelecimento do serviço [1]. Dessa forma, as distribuidoras têm como uma das principais metas reduzir ao máximo o número de interrupções na sua área de serviço. Por fim, a previsão desses serviços emergenciais torna-se cada vez mais indispensável para distribuir de forma eficiente os recursos destinados ao combate de problemas no sistema elétrico e traz como consequência direta a melhoria dos índices de confiabilidade.

A Agência Nacional de Energia Elétrica (ANEEL) estabelece normas e padrões de qualidade, economicidade e continuidade para que as empresas de energia elétrica sigam, visando manter seus clientes abastecidos de energia elétrica. Para garantir que essas normas e padrões sejam cumpridos, a concessionária de energia elétrica deve ter uma estrutura que permita a operação do sistema de distribuição, e que possam ser realizadas operações rotineiras, como intervenções programadas, manobras e manutenção do sistema elétrico. Junto a isso, a empresa concessionária deve ser capaz de detectar falhas no sistema, e corrigi-las no menor tempo possível, de modo que os níveis de desempenho do sistema não sejam afetados [2].

A previsão da demanda é a base do planejamento estratégico da produção, de vendas e finanças de qualquer empresa. Com ela as instituições podem desenvolver seus planos de capacidade, fluxo de caixa, vendas, produção e estoques, mãode-obra, compras, dentre outros. Permite também que os gestores destes sistemas antevejam o futuro e planejem adequadamente suas ações [3].

As previsões de demanda são elaboradas utilizando técnicas qualitativas e quantitativas ou ainda uma mistura de ambas. Métodos quantitativos utilizam dados históricos para prever a demanda em períodos futuros. A previsão da demanda futura requer a construção de modelos matemáticos a partir dos dados disponíveis, ou seja, a partir de dados que descrevem a variação da demanda ao longo do tempo; este grupo de dados é denominado série temporal [4]. Os métodos qualitativos baseiam-se em opiniões de especialistas, apreciação do pessoal de vendas e expectativas dos consumidores e como diferentes indivíduos apresentam preferências e opiniões distintas, esses métodos são vulneráveis à tendências e subjetividades que podem comprometer a confiabilidade de seus resultados [5]. As técnicas que serão implementados nesse trabalho, com exceção do modelo para avaliação dos resultados, serão implementadas através das previsões quantitativas.

Os métodos quantitativos, assim como os qualitativos, são métodos de forecasting ou previsão. Esses métodos são usados para prever os resultados de cursos de ação propostos no planejamento [4. A partir da previsão é possível analisar os resultados aproximados das decisões estratégicas antes mesmo de implementálas. Estas decisões ocorrem na etapa de planejamento, onde a empresa decide as ações que serão tomadas futuramente. Caso os resultados não sejam potencialmente satisfatórios, o processo de previsão e planejamento deve ser repetido.

Este artigo tem como principal objetivo apresentar uma proposta de meto- 
dologia para abordar o problema de previsão de demanda nas empresas, que neste estudo de caso será uma concessionária de energia elétrica, na qual a previsão da demanda terá como objetivo específico analisar e implementar técnicas de previsão a partir de série temporais no intuito de obter o número de ordens de serviço emergenciais que ocorrerão nas próximas 24 horas. Essas técnicas serão comparadas entre si e aquela que apresentar melhores resultados será utilizada no estudo.

\section{FUNDAMENTAÇÃO TEÓRICA}

Planejar é decidir no presente as ações que serão tomadas futuramente, é o processo que descreve atividades necessárias para ir da realidade ao objetivo final estipulado [6]. O primeiro passo no planejamento é, portanto, prever, ou estimar a demanda futura por produtos e serviços e os recursos necessários para pruduzi-los [7].

Gaither e Frazier [7] asseveram que alguns motivos que ilustram a importância de fazer previsões concerne no planejamento de novas instalações, da produção e na programação da força do trabalho.

De acordo com Montgomery [8], outras características importantes do problema de previsão são o intervalo, que é a periodicidade na qual novas previsões são preparadas e o horizonte de previsão, que corresponde ao número de períodos futuros no qual a previsão deverá ser produzida e é comumente descrito pela natureza do problema.

\subsection{TÉCNICAS DE PREVISÃO}

O passo mais importante na modelagem da previsão é a escolha da técnica que melhor se adapte ao problema. Para tanto, é importante entender as características que normalmente são comuns a esse métodos, como: o pressuposto que as causas que influenciaram a demanda continuarão a agir no futuro; que não é possível prever todas as variações aleatórias que ocorrerão no futuro, pois as previsões não são perfeitas; e quanto maior o período de tempo, menor a acuracidade.

É relevante destacar que é possível utilizar diferentes modelos simultaneamente. A figura 1 apresenta alguns modelos de previsão de demanda bem como a forma como são subdivididos.

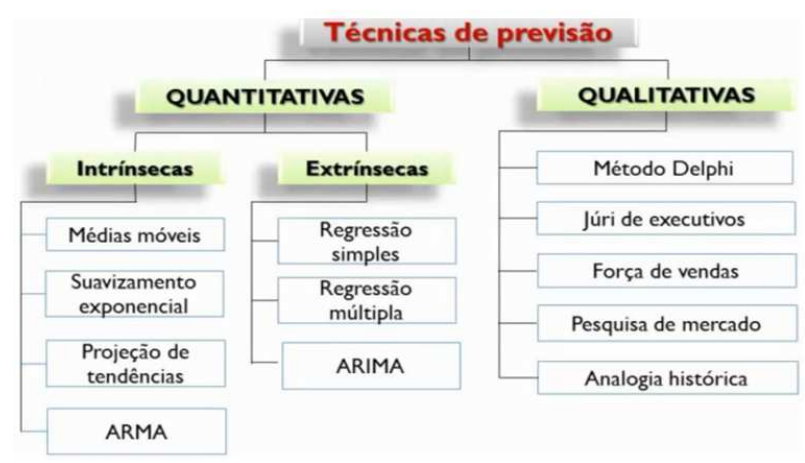

Figura 1: Alguns métodos de previsão de demanda Fonte: [9] 


\subsection{SÉRIES TEMPORAIS}

Uma série temporal é um conjunto de observações sequenciais de uma variável ao longo do tempo, geralmente em intervalos uniformes. Segundo Makridakis [10], a série temporal pode possuir até quatro características conhecidas, conforme elencadas a seguir, com as respectivas definições:

- Média: ocorre quando os valores de uma série oscilam entre um valor médio constante.

- Sazonalidade: ocorre quando existem padrões cíclicos de variação que se repetem em intervalos relativamente constantes de tempo.

- Ciclo: ocorre quando a série apresenta variações ascendentes e descentes não regulares no tempo.

- Tendência: Existe quando a série, de uma maneira geral, apresenta uma tendência ascendente ou descendente quando analisado um longo período de tempo.

Toda variação em uma série temporal que não pode ser classificada nessas quatro características é considerada aleatória, também conhecida como ruído [10]

\subsection{PROCESSOS ESTOCÁSTICOS}

Os modelos utilizados para descrever séries temporais são processos estocásticos, isso é, processos controlados por leis probabilísticas. Seja $T$ um conjunto arbitrário. Um processo estocástico é uma família $Z=\{Z(t), t \in T\}$, tal que, para cada $t \in T, Z(t)$ é uma variável aleatória [11].

Nessas condições, um processo estocástico é uma família de variáveis aleatórias, que por premissa definida num mesmo espaço de probabilidades. O conjunto $T$ é normalmente tomado como o conjunto dos inteiros $\mathbb{Z}=\{0, \pm 1, \pm 2 \ldots\}$ ou o conjunto dos reais $\mathbb{R}$. Também, para cada $t \in T, Z(t)$ será uma variável aleatória real [11].

\subsection{MÉTODOS DE SUAVIZAÇÃO EXPONENCIAL}

Montgomery [8] afirma que um conjunto de dados consiste em duas componentes: sinal e ruído. Sinal representa qualquer padrão causado pela dinâmica intrínseca do processo de onde esses dados foram coletados. De acordo com Montgomery [8], suavização ou alisamento pode ser visto como a técnica para separar ao máximo o sinal do ruído. O suavizador age como filtro para obter uma estimativa do sinal.

\subsubsection{Suavização Exponencial Simples}


(1):

A Suavização Exponencial Simples - SES pode ser descrita conforme a Eq.

$$
\hat{Z}_{t+1}=\alpha Z_{t}+(1-\alpha) \hat{Z}_{t}, \quad t=1, \ldots, N
$$

onde $\overline{Z_{t}}$ é denominado valor exponencialmente suavizado e $\alpha$ é a constante de suavização, $0 \leq \alpha \leq 1$. Quanto mais próximo de 0 for o valor de $\alpha$ mais estável serão as previsões finais, uma vez que a utilização de baixos valores de $\alpha$ implicam que pesos maiores serão dados às observações passadas e, consequentemente, qualquer flutuação aleatória no presente exercerá um peso menor no cálculo da previsão [11]. Quanto mais próximo de 1 for o valor de $\alpha$ mais próximos serão os valores da suavização em relação aos dados originais. Dois casos extremos serão $\alpha=0$ e $\alpha=1$. No primeiro caso a suavização será igual uma constante $\bar{Z}_{0}$. Para $\alpha=1$, teremos $\bar{Z}_{T}=Z_{T}$ e, dessa forma, não haverá suavização.

\subsubsection{Suavização Exponencial de Holt}

A suavização exponencial dupla, também conhecida como suavização exponencial de Holt é uma ferramenta eficaz de previsão desenvolvida por Holt (1957) que expandiu a suavização exponencial simples para dados de série temporal que exibem uma tendência linear [12] A função de previsão do método de Holt é representada pelo conjunto de equações (2), (3) e (4).

$$
\begin{gathered}
L_{t}=\alpha Z_{t}+(1-\alpha)\left(L_{t-1}+T_{t-1}\right) \\
T_{t}=\beta\left(L_{t}-L_{t-1}\right)+(1-\beta) T_{t-1} \\
\hat{Z}_{t+k}=L_{t}+k T_{t}
\end{gathered}
$$

Em que $0 \leq \alpha \leq 1$ e $0 \leq \beta \leq 1$. As equações 2 e 3 fazem estimativa do nível e da tendência e por fim a equação 4 calcula a previsão da série. A interpretação da constante de suavização de tendência $\beta$ é análoga à da constante de suavização do nível $\alpha$.

\subsubsection{Método de Holt-Winters Multiplicativo}

Também chamado de suavização exponencial tripla, o método Holt-Winters é uma expansão do método Holt (1957) desenvolvida por Winter (1960) para aplicar em séries temporais que exibem tendência e sazonalidade. Veríssimo [12] afirma que é um dos métodos mais utilizados para previsão de curto prazo da demanda, devido a sua simplicidade, baixo custo de operação, boa precisão e capacidade de ajustamento automático e rápido a mudanças. Segundo Makridakis[10], as equações (5) a (8) descrevem o modelo:

$$
\begin{gathered}
L_{t}=\alpha \frac{Z_{t}}{S_{t-s}}+(1-\alpha)\left(L_{t-1}+T_{t-1}\right) \\
T_{t}=\beta\left(L_{t}-L_{t-1}\right)+(1-\beta) T_{t-1} \\
S_{t}=\gamma \frac{Z_{t}}{L_{t}}+(1-\gamma) S_{t-s}
\end{gathered}
$$




$$
\hat{Z}_{t+k}=\left(L_{t}+k T_{t}\right) S_{t-s+k}
$$

As equações 5, 6, 7) são utilizadas para estimar o nível, a tendência e a sazonalidade, respectivamente. Os valores das constantes de suavização $\alpha, \beta$ e $\gamma$ são responsáveis pelo controle do nível, tendência e sazonalidade. Por fim, a equação 8 é responsável pela previsão dos valores para os $k$ períodos futuros da série.

Antes de se iniciar o modelo, é necessário obter os valores iniciais de nível, tendência e sazonalidade. As Eq. (9) a (11) calculam esses parâmetros.

$$
L_{s}=\frac{1}{s}\left(Z_{1}+Z_{2}+Z_{3}+\ldots+Z_{s}\right)
$$

Para a tendência, o ideal é utilizar duas estações completas, ou seja, $2 s$ períodos [4].

$$
\begin{gathered}
T_{s}=\frac{1}{s}\left(\frac{Z_{s+1}-Z_{1}}{s}+\frac{Z_{s+2}-Z_{2}}{s}+\ldots+\frac{Z_{s+s}-Z_{s}}{s}\right) \\
S_{1}=\frac{Z_{1}}{L_{s}}, S_{2}=\frac{Z_{2}}{L_{s}}, \ldots, S_{s}=\frac{Z_{s}}{L_{s}}
\end{gathered}
$$

\subsubsection{Método de Holt-Winters Aditivo}

O modelo sazonal aditivo é utilizado quando a amplitude da variação sazonal permanece constante ao longo do tempo. Este modelo é representado pelas equações (12) a (15), a seguir [10]:

$$
\begin{gathered}
L_{t}=\alpha\left(Z_{t}-S_{t-s}\right)+(1-\alpha)\left(L_{t-1}+T_{t-1}\right) \\
T_{t}=\beta\left(L_{t}-L_{t-1}\right)+(1-\beta) T_{t-1} \\
S_{t}=\gamma\left(Z_{t}-L_{t}\right)+(1-\gamma) S_{t-s} \\
\hat{Z}_{t+k}=L_{t}+k T_{t}+S_{t-s+k}
\end{gathered}
$$

Para os componentes de nível e tendência, as fórmulas para calcular os parâmetros iniciais serão as mesmas do multiplicativo, havendo mudança apenas na sazonalidade [10].

$$
S_{1}=z_{1}-L_{s}, S_{2}=z_{2}-L_{s}, \ldots, S_{s}=z_{s}-L_{s}
$$

\subsection{CRITÉRIOS PARA AVALIAÇÃO DA PREVISÃO}

A estimativa da imprecisão pode ser tão útil quanto a própria previsão da demanda esperada. Então, uma boa previsão precisa incluir meios e estimativas de quanto será a variação em torno da média. Essa medida ajuda a entender o risco e permite que sejam tomadas decisões levando em conta a variabilidade presente. Previsão envolve estimar mais do que a demanda esperada, também envolve tentar estimar a incerteza [13.

Para verificar a eficácia do modelo, dados reais passados são comparados às previsões no mesmo período de tempo. Esses erros de previsão, não só mostram 
quão robusto é o modelo, como também fornecem informações a respeito do risco existente na previsão [13]. O erro de previsão para um período $t$ é dado pela Eq. (17):

$$
e_{t}=Z_{t}-\hat{Z}_{t}
$$

Onde $e_{t}$ é o erro da previsão para o período t, $Z_{t}$ é o valor real e $\hat{Z}_{t}$ é o valor previsto pelo modelo. O erro médio $(A E)$ em $n$ períodos encontra-se definido na Eq. (18):

$$
A E=\frac{1}{n} \sum_{i=1}^{n} e_{i}
$$

Porém, como os valores podem ser negativos e positivos, eles tendêm a se anular e dessa forma esse método costuma tender a zero [13]. Dessa forma, para contornar essa situação, é comum a utilização de erros com valores absolutos ou quadráticos [14. Alguns dos critérios de estimativa de erro mais utilizados são Erro Quadrático Médio (MSE) e Média Absoluta dos Erros (MAE) [14].

\subsubsection{Erro Quadrático Médio (MSE)}

No método do erro quadrático médio (Mean Squared Error), pelo fato dos erros serem elevados ao quadrado, o peso dos erros mais distantes da demanda são maiores [14]. A Eq. (19) representa seu cálculo:

$$
M S E_{n}=\frac{1}{n} \sum_{i=1}^{n} e_{i}^{2}
$$

\subsubsection{Erro Absoluto Médio (MAE)}

A média absoluta dos erros (Mean Absolute Error) é muito utilizado nas empresas. Matematicamente. É representado pela Eq. 20 a seguir:

$$
M A E_{n}=\frac{1}{n} \sum_{i=1}^{n}\left|e_{i}\right|
$$

onde $\left|e_{i}\right|$ é o módulo de $e_{i}$. Como nesse método se utiliza o módulo dos termos de erro, essa forma de medição de erro consegue capturar os desvios positivos e negativos entre a previsão e a demanda real [14].

\section{ESTUDO DE CASO}

De acordo com o Grupo Energisa [15, a Energisa Tocantins Distribuidora SA (ETO) foi a primeira concessionária estadual de energia a ser privatizada no Brasil e é a única distribuidora de energia elétrica do estado do Tocantins, cobrindo uma área de $277.621 \mathrm{~km}^{2}$, aproximadamente $3,3 \%$ do território nacional, beneficiando uma população estimada em 1,5 milhão de habitantes, distribuídos em 139 munucípios, o que corresponde a 546.422 clientes atendidos. Hoje, a ETO é a maior 
empresa privada do Tocantins.

Esse trabalho apresenta um estudo utilizando dados da Energisa Tocantins Distribuidora SA (ETO). Nesse material pode ser encontrado o volume de ordens de serviço da região sul do estado de Tocantins, e também parâmetros importantes como sua localização, horário de solicitação, horário de conclusão, causa técnica, dentre outros. Os dados foram disponibilizados em função do projeto de pesquisa e desenvolvimento que vem sendo executado pela UFF SIDEC - Sistema Inteligente para Dimensionamento de Equipes de Campo e se iniciam em maio de $2016 \mathrm{com}$ término em setembro do mesmo ano [16].

Na base de informações, existem 39 municípios com parâmetros disponíveis, porém, como esse trabalho necessita de apenas uma localidade, o estudo será realizado com os dados do município de Gurupi.

\subsection{QUALIDADE DO FORNECIMENTO DE ENERGIA}

Uma ordem de serviço (OS) é criada quando o cliente liga para a central de atendimento (call center) da empresa e o atendente registra a ocorrência. Se o serviço é caracterizado como emergencial, uma OS é imediatamente processada e enviada para o Centro de Operações (COD) que rapidamente transmite essa ordem de serviço para o veículo mais próximo do local da ocorrência, para ser atendida no menor tempo possível. Porém, caso o serviço seja considerado como comercial, a OS gerada é enviada para o COD, mas entra na fila de espera [17]. O número de ocorrências está diretamente relacionado com as condições climáticas. Altas temperaturas contribuem para o aparecimento de problemas de mal contato como também de desarme e queima de equipamentos [18].

A principal missão das empresas de distribuição de energia elétrica é proporcionar ao cliente um fornecimento de energia contínuo e com qualidade. Além do aspecto de atendimento às expectativas dos consumidores, as distribuidoras de energia têm que cumprir as exigências da regulação específica do setor elétrico, estabelecidas pela Agência Nacional de Energia Elétrica (ANEEL) [18]. O módulo 8 do Procedimento de Distribuição de Energia Elétrica no Sistema Elétrico Nacional (PRODIST) é utilizado pela ANEEL com o objetivo de estabelecer os procedimentos relativos à qualidade da energia elétrica (QEE), abordando a qualidade do produto, a qualidade do serviço prestado e a qualidade do tratamento de reclamações [19]. No módulo 8 do PRODIST [19], a ANEEL estabelece 4 indicadores de tempo de atendimento referente às ocorrências emergenciais. Tempo Médio de Preparação (TMP), Tempo Médio de Deslocamento (TMD), Tempo Médio de Execução (TME) e Tempo Médio de Atendimento a Emergências (TMAE). A forma de se calcular cada um desses indicadores encontra-se, respectivamente nas Eq. (21) a (24):

$$
\begin{aligned}
& T M P=\frac{\sum_{i=1}^{n} T P(i)}{n} \\
& T M D=\frac{\sum_{i=1}^{n} T D(i)}{n}
\end{aligned}
$$




$$
\begin{gathered}
T M E=\frac{\sum_{i=1}^{n} T E(i)}{n} \\
T M A E=T M P+T M D+T M E
\end{gathered}
$$

\subsection{CRITÉRIOS DE ANÁLISE DOS MODELOS}

Para avaliar qual método será utilizado no estudo de caso, será adotado um horizonte de previsão de 30 dias, ou seja, os modelos serão treinados do período de maio a agosto e serão a base para a previsão de todo o mês de setembro. Esses dados serão comparados com os dados reais e o melhor modelo será aquele que apresentar o menor erro absoluto médio (MAE). O MAE é superior aos demais erros no caso estudado por ser menos afetado por erros anomalamente extremos chamados "pontos singulares" ou "outliers" [14]. Na etapa de modelagem foi utilizada a biblioteca statsmodels. Os valores das constantes de suavização já são otimizados pela biblioteca para todos os casos. A figura 2 apresenta a série temporal com as ordens de serviço emergenciais pelo número de horas, entre os períodos de maio a setembro de 2016.

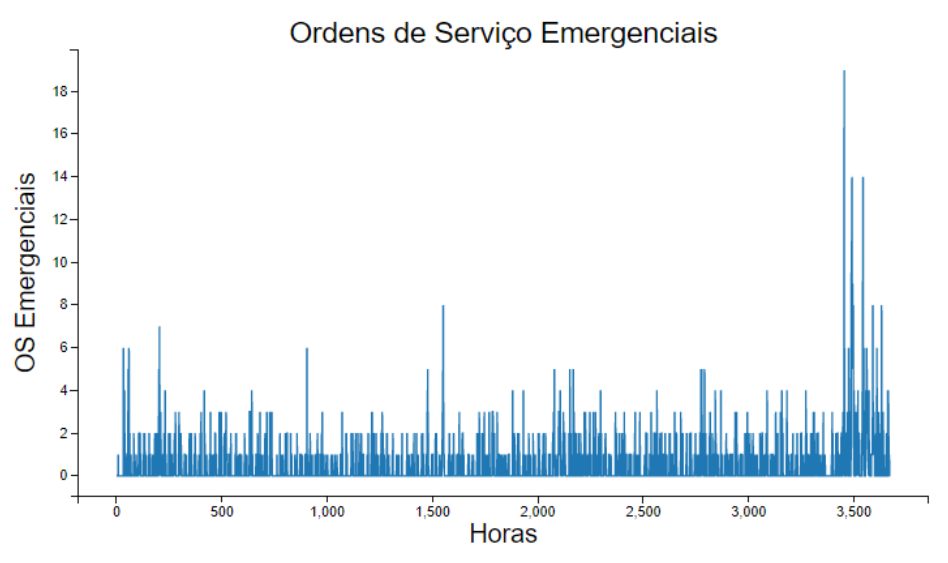

Figura 2: Ordens de serviço emergenciais da ETO Fonte: Reprodução própria

\subsection{ESCOLHA DO MODELO DE PREVISÃO}

Após a implementação de cada modelo, é possível compará-los para verificar qual apresentou o melhor resultado e assim, utilizá-lo para analisar cada um dos 30 dias do mês de setembro individualmente. A tabela 1 apresenta todos os métodos e seus respectivos erros absolutos médios. 


\begin{tabular}{|c|c|}
\hline Métodos & MAE \\
\hline SES & 0,92662 \\
\hline Holt & 0,93361 \\
\hline Holt-Winters aditivo & 0,84845 \\
\hline Holt-Winters multiplicativo & 0,84742 \\
\hline
\end{tabular}

Tabela 1: Métodos de previsão e seus respectivos erros absolutos médios. Fonte: Reprodução própria

A partir das informações da tabela 1 pode-se perceber que os modelos de Holt-Winters apresentaram os melhores resultados. Dessa forma, a escolha do modelo se dá entre o método multiplicativo e o aditivo. Apesar de ambos apresentarem valores muito próximos, no modelo multiplicativo o Erro Absoluto Médio (MAE) foi inferior, dessa forma será utilizado para a modelagem do problema. A figura 3 apresenta essa previsão.

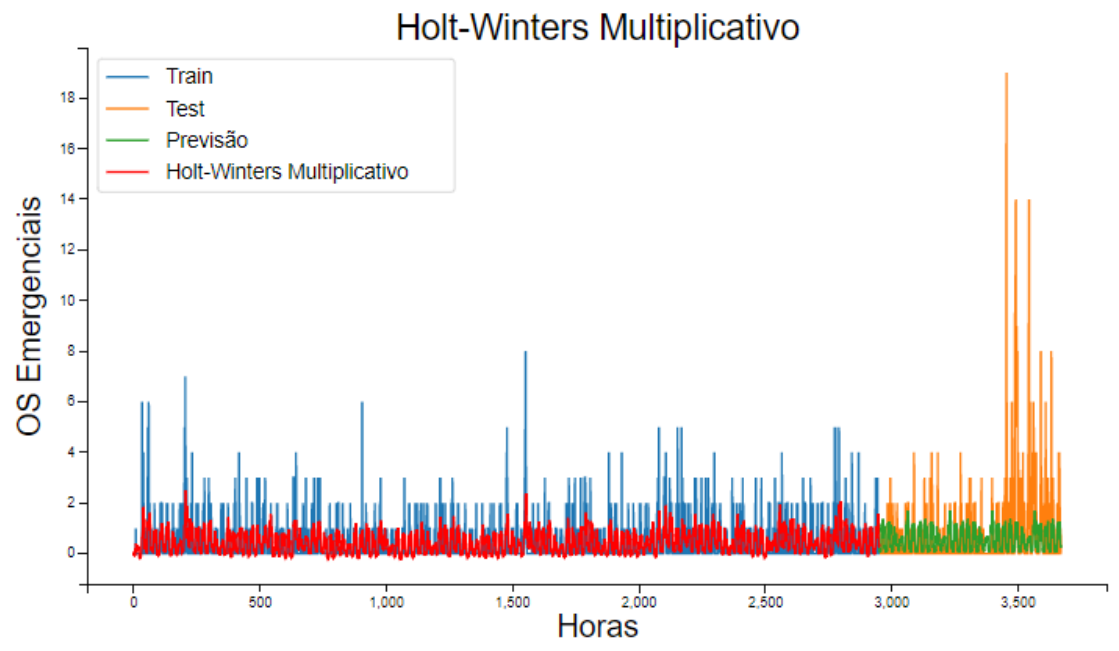

Figura 3: Previsão por suavização exponencial de Holt-Winters multiplicativo no período de setembro. Fonte: Reprodução própria

\section{RESULTADOS}

Nesta seção será realizada a modelagem da principal previsão do trabalho utilizando o método escolhido na etapa anterior. O problema a ser modelado é a previsão da demanda de ordens de serviço emergenciais para as próximas 24 horas através dos dados históricos. Com esse conhecimento, a concessionária poderá se preparar no dia anterior da forma mais conveniente possível e diariamente possuirá previsões atualizadas. Nesse estudo de caso, o modelo utiliza os dados históricos de maio a agosto de 2016 para prever a demanda horária das ordens de serviço emergenciais do dia 1 de setembro do mesmo ano. Ele irá começar às 00:00:00 do dia primeiro de setembro e fará a previsão das próximas 24 horas utilizando todos os dados históricos. Após 24 horas, os valores previstos serão comparados com os dados reais e o erro será calculado. Esses dados reais serão acrescentados aos dados 
históricos e a previsão se repetirá para o dia seguinte. Esse processo persiste até a previsão horária do último dia de setembro.

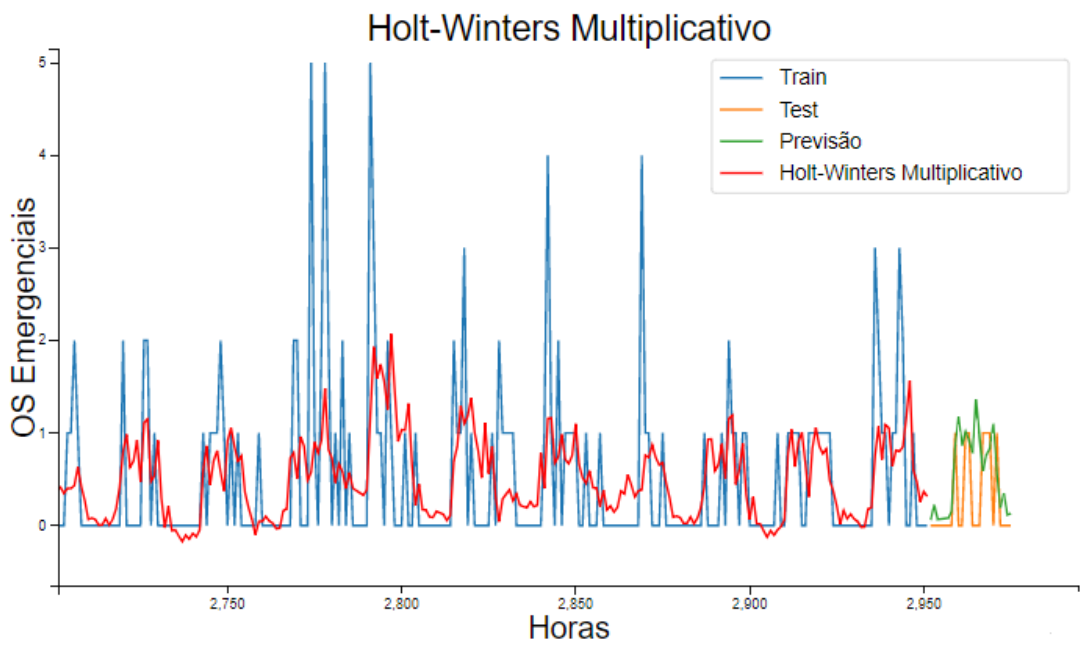

Figura 4: Previsão por suavização exponencial de Holt-Winters multiplicativo no dia 1 de setembro. Fonte: Reprodução própria

\subsection{Avaliação comparativa de desempenho do modelo}

Para verificar se o modelo de previsão desenvolvido nesse trabalho apresenta resultados que geram impacto para a empresa, sua performance será comparada com a de um modelo simples, porém robusto. O método consiste em calcular a média aritmética dos dados com o mesmo dia da semana e mesma hora e utilizar esse valor como previsão para a semana seguinte. Enquanto o modelo utilizando HoltWinters utiliza apenas a sazonalidade semanal, o critério de avaliação com média aritmética leva em conta tanto a zonalidade semanal, por relacionar dias da semana semelhantes, como a sazonalidade diária, por relacionar horários semelhantes.

O período da série no qual ocorrerão as comparações será de 7 dias, compreendidos entre os dias 5 e 11 de setembro de 2016. Serão utilizados dados históricos das 18 semanas passadas. O período foi escolhido de forma arbitrária de 18 semanas corresponde a todas as semanas anteriores ao período estudado. Os resultados de ambos os modelos serão comparados dia a dia e os critérios para determinar o modelo mais eficaz serão erro absoluto diário e maior número de erros horários inferiores ao do modelo concorrente.

Para aperfeiçoar a análise, foi criado um critério que apresenta em quantas horas do dia cada modelo atinge resultados superiores ao outro. Um modelo que tenha menores erros na maioria das horas do dia e tenha maior erro médio pode ser interessante dependendo da situação do usuário. Principalmente no caso desse modelo apresentar menores erros em horários que a empresa prioriza.

Na figura 5 podem ser vistos 2 gráficos distintos. O de acima apresenta os erros absolutos horários de cada critério. O segundo gráfico é gerado através da diferença entre o erro da média e o erro do modelo de Holt-Winters, todos os 
valores superiores a zero indicam que o erro de Holt-Winters é menor do que o erro da média.
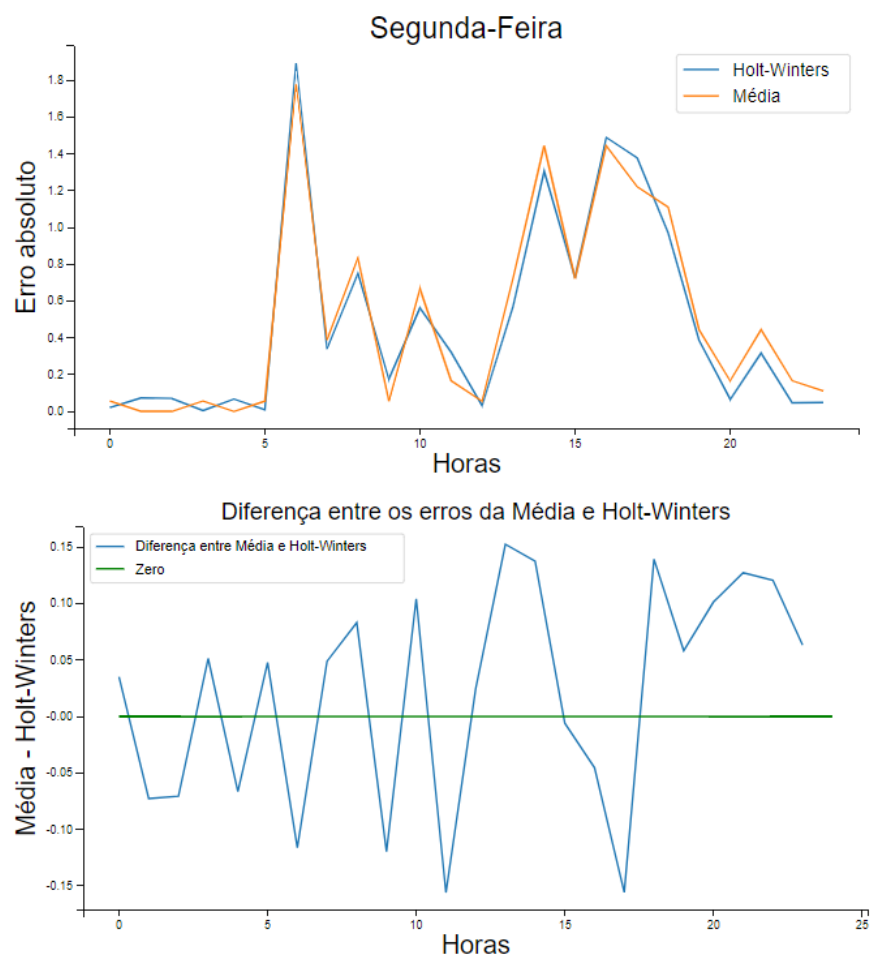

Figura 5: Resultado da comparação pelo critério do maior número de erros horários para segunda-feira. Fonte: Reprodução própria

A tabela 2 apresenta os resultados dos 7 dias estudados. Nela são apresentados os erros absolutos diários e o número de horas que determinado modelo teve menor erro absoluto para cada dia da semana. O número de erros horários de menor magnitude serão chamados de "vitórias".

\begin{tabular}{|c|c|c|c|c|}
\hline & \multicolumn{2}{|c|}{ Média } & \multicolumn{2}{c|}{ Holt-Winters } \\
\hline & Erro Absoluto & Vitórias & Erro Absoluto & Vitórias \\
\hline Segunda-feira & 12,1111 & 9 & 11,6238 & 15 \\
\hline Terça-feira & 12,8889 & 13 & 13,2920 & 11 \\
\hline Quarta-feira & 8,1667 & 20 & 9,6148 & 4 \\
\hline Quinta-feira & 13,1667 & 8 & 12,3124 & 16 \\
\hline Sexta-feira & 16,0556 & 11 & 16,3936 & 13 \\
\hline Sábado & 11,6111 & 19 & 16,5088 & 5 \\
\hline Domingo & 7,9444 & 21 & 8,8688 & 4 \\
\hline
\end{tabular}

Tabela 2: Métodos de previsão e seus respectivos erros absolutos médios. Fonte: Reprodução própria

A partir dos resultados obtidos na comparação dos modelos pode-se perceber que o método de Holt-Winters apresenta eficácia inferior à média dos dias da semana. As razões principais dessa situação ocorrer são a existência de alta varia- 
bilidade e principalmente o fato da série ter duas sazonalidades e a média semanal levar em consideração ambas.

Para contornar o problema da alta variabilidade da série, uma nova previsão ocorrerá a partir do logarítmo natural da série e, em seguida, tanto os dados históricos quanto a previsão serão retornados aos valores normais inserindo a base exponencial de Euler (e). Esse método será utilizado pois o logarítmo natural tem a capacidade de atenuar a série, dessa forma, reduz a influência de dados discrepantes [20]. A tabela 3 demonstra que Holt-Winters multiplicativo utilizando logarítmo apresenta a melhor perfomance entre todos os modelos.

\begin{tabular}{|c|c|c|c|}
\hline$\alpha$ & $\beta$ & $\gamma$ & MAE \\
\hline 0,053079 & 0,0 & 0,0 & 0,82680 \\
\hline
\end{tabular}

Tabela 3: Melhores constantes de suavização $\alpha, \beta$ e $\gamma$ e seu respectivo $M A E$. Fonte: Reprodução própria

A tabela 4 apresenta a comparação dos resultados da média semanal e do modelo de Holt-Winters multiplicativo após aplicação da ferramenta matemática do logarítmo.

\begin{tabular}{|c|c|c|c|c|}
\hline & \multicolumn{2}{|c|}{ Média } & \multicolumn{2}{c|}{ Holt-Winters } \\
\hline & Erro Absoluto & Vitórias & Erro Absoluto & Vitórias \\
\hline Segunda-feira & 12,1111 & 10 & 11,9096 & 14 \\
\hline Terça-feira & 12,8889 & 13 & 12,6844 & 11 \\
\hline Quarta-feira & 8,1667 & 12 & 8,0392 & 12 \\
\hline Quinta-feira & 13,1667 & 8 & 10,9325 & 16 \\
\hline Sexta-feira & 16,0556 & 11 & 16,0573 & 13 \\
\hline Sábado & 11,6111 & 11 & 11,3853 & 13 \\
\hline Domingo & 7,9444 & 6 & 6,7263 & 18 \\
\hline
\end{tabular}

Tabela 4: Métodos de previsão e seus respectivos erros absolutos médios. Fonte: Reprodução própria

A tabela 4 mostra que o erro absoluto após a aplicação do logarítmo só perde para a média semanal em um único dia e por uma pequena margem. Com relação ao número de erros horários, o modelo também perde apenas em um dia comparado ao método de teste.

\section{CONCLUSÕES}

O estudo apresentado mostrou a importância da previsão da demanda no ambiente empresarial bem como técnicas para implementá-la. Para o caso da Energisa Tocantins (ETO) foi visto que o modelo de Holt-Winters superou o método da média aritmética que utilizava ambas as sazonalidades semanais e diárias.

A explicação para a melhora nos resultados através da aplicação do logarítmo é que assim como na suavização exponencial, sua aplicação atenua as oscilações da série, e dessa forma, facilita a descoberta de padrões pelo modelo. 
As ordens de serviço emergenciais são as principais causadoras de quedas de energia e causam prejuízos financeiros e patrimoniais para a concessionária a medida que danificam rede de distribuição. O objetivo dessa previsão para a concessionária é, através do conhecimento aproximado de acontecimentos futuros, maximizar a eficácia das tomadas de decisão e, dessa forma, reduzir custos e melhorar a qualidade do serviço para a população.

A utilização diária da previsão pela concessionária pode reduzir custos de mobilização de pessoal, custos de alocação e mobilização dos equipamentos necessários para o atendimento dessas ordens, como também reduzir indicadores de duração da interrupção de energia como o DEC. A utilização da previsão nos períodos de médio e longo prazo ainda auxiliam na resolução do problema de alocação das bases.

Como as ordens emergenciais são quase em sua totalidade causadas por fenômenos climáticos, a previsão com horizonte de alguns meses não capta de forma eficiente seus padrões. Essa situação foi a principal razão pela qual o modelo não apresentou resultados ainda melhores. Mesmo existindo duas sazonalidades na série utilizada no estudo, os dados tinham caráter altamente aleatório e poucos padrões a serem assimilados pelo modelo.

Seria interessante o estudo da previsão com dados em intervalos mensais e horizonte de alguns anos. Dessa forma, o modelo poderia captar a sazonalidade anual das estações, reconhecendo assim os períodos secos e chuvosos. A previsão de Holt-Winters se torna mais robusta a medida que a sazonalidade se torna mais evidente, o que muito provavelmente faria com que o modelo em períodos mais longos gerassem melhores previsões. Outro ponto a ser explorado seria a utilização das técnicas qualitativas em conjunto com os modelos quantitativos de previsão de séries temporais, o que maximizaria a eficácia dos modelos e enriqueceria o estudo.

\section{AGRADECIMENTOS}

O presente trabalho foi realizado com apoio da Coordenação de Aperfeiçoamento de Pessoal de Nível Superior - Brasil (CAPES) - Código de Financiamento 001.

\section{REFERÊNCIAS BIBLIOGRÁFICAS}

[1] TRONCHONI, A. B. Identificação de causas de desligamentos não programados em redes de distribuição. Dissertação (Mestrado em Engenharia Elétrica) Pontifícia Universidade Católica do Rio Grande do Sul, março 2008. Disponível em: <http://repositorio.pucrs.br/dspace/bitstream/10923/3225/1/000403015Texto\%2BCompleto-0.pdf>. Acesso em: 20 de setembro de 2018, 15:30:00. 2

[2] (ELETROBRAS), C. E. brasileiras. Manutenção e Operação de Sistemas de Distribuição. 4. ed. [S.l.]: Campus, 1982. 2

[3] ZANELlA, C.; VIEIRA, V.; BARICHELLO, R. Previsão de demanda: um estudo de caso em uma agroindústria de carnes do oeste catarinense. GEPROS, 2015. Disponível em: 
<https://revista.feb.unesp.br/index.php/gepros/article/view/1310>.

Acesso em: 20 de setembro de 2018, 18:00:00. 2

[4] PELLEGRINI, F. R. Metodologia para implementação de sistemas de previsão de demanda. Dissertação (Mestrado em Engenharia de Produção) Universidade Federal do Rio Grande do Sul, setembro 2000. Disponível em: $<$ http://www.producao.ufrgs.br/arquivos/publicacoes/Fernando\%20R\%20Pellegrini.pdf $>$. Acesso em: 20 de setembro de 2018, 17:00:00. 2, 6

[5] KRAJEWSKI, L. J.; RITZMAN, L. P. Operations Management, Strategy and Analysis. 5. ed. [S.l.]: Addison-Wesley, 1999. 2

[6] MARTINS, P.; LAUGENI, F. Administração da produção. 2. ed. [S.l.]: Saraiva, 2005. 3

[7] GAITHER, N.; FAZIER, G. Administração da produção e operações. 8. ed. [S.l.]: Pioneira Thomsom Learning, 2002. 3

[8] MONTGOMERY, D.; JENNINGS, C.; KULAHCI, M. Introduction to time series analysis and forecasting. [S.l.]: Wiley, 2008. 3, 4

[9] MARTINS, V. et al. Previsão de demanda de passageiros para os aeroportos de fortaleza, brasília, são paulo e rio de janeiro durante a realização da copa do mundo de 2014. CONEPRO, 2014. 3

[10] MAKRIDAKIS, S. G.; WHEELWRIGHT, S. C.; HYNDMAN, R. J. Forecasting: Methods and Applications. 3. ed. [S.l.]: John Wiley, 1997. 4, 5, 6

[11] MORETTIN, P. A.; TOLOI, C. M. Análise de séries temporais. 3. ed. Edgard Blucher LTDA, 2004. Disponível em: $<$ https://www.passeidireto.com/arquivo/26097461/morettin-analise-de-seriestemporais >. Acesso em: 25 de outubro de 2018, 13:40:00. 4, 5

[12] VERÍSSIMO, A. J. e. a. Métodos estatísticos de suavização exponencial holt-winters para previsão de demanda em uma empresa do setor metal mecânico. Revista Gestão Industrial, 2012. Disponível em: $<$ https://periodicos.utfpr.edu.br/revistagi/article/view/1378>. Acesso em: 25 de outubro de 2018, 15:20:00. 5

[13] BLOCHER, J. D. et al. Forecasting: Including an introduction to forecasting using the sap $\mathrm{r} / 3$ system. Indiana University Kelley School of Business, 2004. Disponível em: $<$ https://kelley.iu.edu/mabert/e730/Forecasting_February_2004.pdf $>$. Acesso em: 25 de novembro de 2018, 21:15:00. 6, 7

[14] HALLAK, R.; FILHO, A. J. P. Metodologia para análise de desempenho de simulações de sistemas convectivos na região metropolitana de são paulo com o modelo arps: sensibilidade a variações com os esquemas de advecção e assimilação de dados. Revista Brasileira de Meteorologia, 2010. Disponível em: <http://www.scielo.br/pdf/rbmet/v26n4/a09v26n4.pdf>. Acesso em: 15 de maio de 2019, 15:00:00. 7, 9 
[15] ENERGISA. Relatório anual de sustentabilidade socioambiental 2014. [S.1.], 2014. Disponível em: <http://grupoenergisa.com.br/Documents/relatoriossocioambientais/RS\%20Energisa\%202014.pdf $>$. Acesso em: 12 de dezembro de 2018, 21:30:00. 7

[16] JUSTO, H. Alocação de bases de equipes de campo utilizando otimização bioinspirada. Dissertação (Graduação em Engenharia Elétrica) — Universidade Federal Fluminense, 2018. 8

[17] MAGRO, M. A. de B. Dimensionamento de equipes baseado em modelos de previsão, simulação e alocação: Caso de uma empresa do setor elétrico. Dissertação (Mestrado em Engenharia de Produção) - Universidade Federal do Rio Grande do Sul, dezembro 2003. Disponível em: <https://www.lume.ufrgs.br/bitstream/handle/10183/4564/000457665.pdf?se quence=1>. Acesso em: 29 de novembro de 2018, 09:00:00. 8

[18] AMORIM, M. L. F. Otimização de atendimentos de emergência em redes de distribuição de energia elétrica. Dissertação (Mestrado em Computação) - Universidade Federal Fluminense, julho 2010. Disponível em: <http://www2.ic.uff.br/PosGraduacao/Dissertacoes/471.pdf>. Acesso em: 29 de novembro de 2018, 11:40:00. 8

[19] ANEEL. Procedimentos de Distribuição de Energia Elétrica no Sistema Elétrico Nacional - Módulo 8 - Qualidade de Energia Elétrica. [S.1.], 2017. Disponível em: <http://www.aneel.gov.br/documents/656827/14866914/Módulo_8Revisão_10/2f7cb862-e9d7-3295-729a-b619ac6baab9i. Acesso em: 29 nov. 2018>. 8

[20] GAUDEnCIO, J. H. D. et al. Previsão de dados de séries temporais dos pibs brasileiro e americano através do modelo arima. XXXIV ENCONTRO NACIONAL DE ENGENHARIA DE PRODUCAO, 2014. Disponível em: $<$ http://www.abepro.org.br/biblioteca/enegep2014_TN_STP_200_134_23519.pdf>. Acesso em: 25 de novembro de 2018, 21:15:00. 13 